\title{
Rind Thickness of Watermelon Cultivars for Use in Pickle Production
}

\author{
Gabriele Gusmini, \\ Jonathan R. Schultheis, and \\ Todd C. Wehner ${ }^{1}$
}

AdDitional INDEX wORds. Citrullus lanatus, Cucurbitaceae, brining, vegetable breeding

SumMary, Salted and sweet watermelon rind pickles are commonly produced in North America, Europe, and Asia using traditional recipes. Homeowners and small industries use the leftover watermelon crop, especially from cultivars having thick and crisp rind, to produce pickles. Recently, we classified rind thickness for a set of obsolete and heirloom cultivars used by home gardeners and heirloom collectors in the United States. In this study, we used elite cultivars for growers interested in high yield, fruit quality, adaptability, and disease resistance. The objective of this study was to classify modern cultivars (nine inbreds and $103 \mathrm{~F}_{1}$ hybrids) of watermelons available to growers for use in production of watermelon rind pickles. Based on the data, cultivars were divided into three groups of rind thickness and categorized according to pedigree (inbred or $F_{1}$ hybrid), fruit type (seeded or seedless), and flesh color (red, orange, or yellow). Most of the cultivars tested (109 of 112) had rind thicker than $10 \mathrm{~mm}$ and could be used for pickle production.

$\mathrm{W}$ atermelon (Citrullus lanatus) production in the U.S. in 1998 to 2003 averaged 1.77 million $\mathrm{Mg}$ (39.1 million cwt) of marketable fruit per year from 75,000 ha $(185,322$ acres $)$ planted $(89 \%$ harvested), with a yield of $26.83 \mathrm{Mg} \cdot \mathrm{ha}^{-1}$ (239.4 cwt/acre) per year (USDAARS, 2004). The market value has been

Department of Horticultural Science, North Carolina State University, Raleigh, NC 27695-7609.

The authors gratefully acknowledge the technical assistance of Dennis E. Adams.

${ }^{1}$ To whom reprint requests should be addressed. E-mail address: Todd_Wehner@NCSU.Edu 
stable in the last 5 years, with an average total value of $\$ 280$ million. Most of the watermelons have been marketed for use as fresh fruit, both whole and pre-cut. Nevertheless, watermelon fruit can be used also for production of rind pickles.

Homeowners and small industries use the leftover watermelon crop, especially from cultivars having thick and crisp rind, to produce pickles. Salted and sweet watermelon pickles are commonly produced in North America, Europe, and Asia based on traditional recipes (Gusmini and Wehner, 2003a; Simonne et al., 2003). Recently, some traditional American recipes were compared for their efficacy, food safety, quality, and suitability for industrial production of watermelon rind pickles (Simonne et al., 2003). Their major concern was to determine the correct balance of brine acidity that would avoid the germination of spores of Clostridium botulinum, responsible for the deadly disease botulism, without damaging the delicate structure of the watermelon rind. They concluded that a brine $\mathrm{pH}$ lower than 4.6 should be used in industrial production and that homeowners should avoid recipes that do not include vinegar in the brine.

The watermelon fruit consists of the exocarp, mesocarp, and endocarp. The endocarp is the seed-containing part that is consumed as food, and the mesocarp and exocarp are usually referred to as the rind. The rind is used for making pickles after removing the thin exocarp, leaving the crisp, white mesocarp. Some recipes suggest that a thin layer of colored endocarp be left with the white mesocarp, possibly to add some watermelon flavor to the final product. The most important characteristics of the rind to be suitable for watermelon pickle production is thickness: usually the rind is cut into cubes of 10 to $20 \mathrm{~mm} \mathrm{(0.39}$ to 0.79 inch) per side.

The inheritance of rind thickness in watermelon was studied in India in 1975 using three crosses of six generations each. Based on these crosses, rind thickness in watermelon was described as a quantitative trait with a larger contribution to its inheritance from additive than dominant genetic components (Sharma and Choudhury, 1988). More recently, differences in rind thickness due to ploidy have been presented (Abd el Hafez, 1982). Although far from conclusive, this study indicated a positive correlation between rind thickness and ploidy. The rind is derived from tissues of the ovary, as is the rest of the watermelon fruit. In induced autopolyploid plants such as triploid and tetraploid watermelons, the cells of the flower, as well as the flower itself, are larger than those of the diploid. Therefore, the rind is expected to be thicker in tetraploid and triploid than in diploid fruit.

Recently, we classified rind thickness for a set of obsolete and heirloom cultivars used by home gardeners and heirloom collectors in the U.S. 'Carolina Cross \#183', 'Cobbs Gem', 'Florida Favorite', 'Garrisonian', 'Malali', 'Moon \& Stars', 'Navajo Sweet', 'Smokylee', 'Stone Mountain', 'Tendersweet Orange Flesh', 'Tom Watson,' and 'Weeks NC Giant' had thick rind (more than $20 \mathrm{~mm}$ ). 'Calhoun Gray', 'Charleston Gray', 'Dixielee', 'Fairfax', 'Georgia Rattlesnake', 'Mardi Gras', 'Mountain Hoosier', 'Regency', 'Sun Gold', 'Tendergold', and 'Tastigold' had thinner rind, but still suitable for rind pickle production [15-20 mm (0.59-0.79 inch)] (Gusmini and Wehner, 2003b, 2004). However, most of those cultivars were not adequate for growers seeking high yield, fruit quality, adaptability, and disease resistance.

The objective of this study was to classify modern cultivars of watermelons available to growers for use in production of watermelon rind pickles.

\section{Materials and methods}

For this study, we used data for rind thickness obtained from eight North Carolina watermelon cultivar trials conducted from 1995 through 2002 at the Central Crops Research Station at Clayton, Horticultural Crops Research Station at Clinton, Cunningham Research Station at Kinston, and a grower's field in Chowan County (Schultheis, 1995, 1996, 1997, 1998, $1999,2000,2001,2002)$. Each of the 112 cultivars (nine inbreds and 103 $\mathrm{F}_{1}$ hybrids) included in our analysis were grown in one to seven trials $($ mean $=2)$.

Each trial had a randomized complete-block design with one location, (Clayton, Clinton, Kinston, or Chowan County) 1 year (1995 to 2002), and three replications. In each trial, the seedlings were produced in LE 1803 plastic trays in the greenhouse of the
Department of Horticultural Science at North Carolina State University, Raleigh. An artificial soilless growing medium was used. The trays were moistened to capacity $24 \mathrm{~h}$ prior to seeding. After seeding, the trays were held in a germination chamber [temperature $\left.=29.4^{\circ} \mathrm{C}\left(85^{\circ} \mathrm{F}\right)\right]$ until $10 \%$ of the seeds germinated. The trays were then transferred to the greenhouse until full emergence and held in an open cold frame at the field site for acclimation for 1 to 2 weeks prior to transplanting. The seedlings were transplanted on raised beds covered with black polyethylene mulch, and irrigated using a drip system. Field preparation varied depending on soil type at the different locations and in each year. In all trials, the soil was fumigated prior to bedding with 1,3-dichloropropene and chloropicrin (Telone C-17; Dow AgroSciences Canada Inc., Calgary, Alta., Canada). Fertilizers were broadcast and disk incorporated during field preparation. Additional fertilizer was injected through the irrigation system as required by each trial (Sanders, 2004).

In each trial, rind thickness was measured in millimeters with a ruler from the outer edge of the fruit to the boundary between the white mesocarp and colored endocarp for each harvest, with up to five fruit per plot randomly chosen among the mature ones. Fruit were determined to be ripe by looking for a dried tendril nearest the fruit, a light-colored ground spot, and a dull sound of the fruit when thumped (Maynard, 2001). Fruit were cut longitudinally (from the stem to the blossom end) and rind thickness was measured at mid-length of the fruit section. Means by trial for each cultivar were used to generate overall means for rind thickness. The overall least significant difference [LSD $(\alpha=$ $0.05)$ ] for rind thickness was calculated based on the LSD presented for each trial. We classified the cultivars based on pedigree (inbred or $\mathrm{F}$ hybrid), fruit type (seeded or seedless), and flesh color (red, orange, or yellow). We divided the cultivars based on their overall mean rind thickness into three groups as follows:

- Group 1: rind thickness $>19 \mathrm{~mm}$ (0.75 inch)

- Group 2: rind thickness $=10-19 \mathrm{~mm}$ (0.30-0.75 inch)

- Group 3: rind thickness $<10 \mathrm{~mm}$ (0.39 inch) 
Table 1. Mean rind thickness for the watermelon cultivars evaluated in North Carolina in replicated trials conducted between 1995 and 2002, along with type, flesh, and rind color descriptors.

\begin{tabular}{|c|c|c|c|c|c|c|c|}
\hline $\begin{array}{l}\text { Cultivar } \\
\text { name }\end{array}$ & $\begin{array}{c}\text { Cultivar } \\
\text { type }\end{array}$ & $\begin{array}{c}\text { Seed } \\
\text { source }^{y}\end{array}$ & $\begin{array}{c}\text { Fruit } \\
\text { type }\end{array}$ & $\begin{array}{l}\text { Flesh } \\
\text { color }\end{array}$ & $\begin{array}{l}\text { Rind } \\
\text { color }^{\mathrm{x}}\end{array}$ & $\begin{array}{c}\text { Years } \\
\text { of trial }^{w}\end{array}$ & $\begin{array}{l}\text { Rind } \\
\text { thickness } \\
(\mathrm{mm})^{\mathrm{v}}\end{array}$ \\
\hline \multicolumn{8}{|c|}{ Group 1: rind thickness $>19 \mathrm{~mm}(0.75 \mathrm{inch})$} \\
\hline Fantastik & $\mathrm{Fl}$ & A. \& C. & Seedless & Red & MD & 1 & 24 \\
\hline Sweetheart & $\mathrm{Fl}$ & Zer. Ged. & Seedless & Red & MS & 1 & 24 \\
\hline Triple Crown & $\mathrm{Fl}$ & Seedw. & Seedless & Red & MD & 1 & 24 \\
\hline Jubilee & Fl & Will. & Seeded & Red & NR & 1 & 23 \\
\hline Slice-N-Serve 830 & $\mathrm{Fl}$ & Southw. & Seedless & Red & MD & 2 & 23 \\
\hline Summer Sweet 5032 & Fl & A. \& C. & Seedless & Red & WD & 1 & 23 \\
\hline Trillion & $\mathrm{Fl}$ & A. \& C. & Seedless & Red & MD & 2 & 23 \\
\hline Triple Prize & Fl & Seedw. & Seedless & Red & $\mathrm{MD}$ & 1 & 23 \\
\hline Tri-X-Carousel & Fl & Syng. & Seedless & Red & WD & 2 & 23 \\
\hline Arriba & Fl & Holl. & Seeded & Red & MD & 1 & 22 \\
\hline Charleston Gray & OP & Will. & Seeded & Red & GR & 1 & 22 \\
\hline Desert King & $\mathrm{OP}$ & Will. & Seeded & Salmon yellow & LS & 1 & 22 \\
\hline Elation & Fl & D. Palm. & Seeded & Red & MD & 1 & 22 \\
\hline Mara & $\mathrm{Fl}$ & Uni. Gen. & Seeded & Red & WD & 1 & 22 \\
\hline Montreal & $\mathrm{Fl}$ & Suns. & Seeded & Red & WD & 2 & 22 \\
\hline Royal Jubilee & Fl & Sem. & Seeded & Red & $\mathrm{NR}$ & 1 & 22 \\
\hline Starbrite & $\mathrm{Fl}$ & Sem. & Seeded & Red & NR & 5 & 22 \\
\hline Summer Flavor 800 & Fl & A. \& C. & Seeded & Red & WD & 2 & 22 \\
\hline Athens & $\mathrm{Fl}$ & Suns. & Seeded & Red & WD & 2 & 21 \\
\hline Cooperstown & Fl & Sem. & Seedless & Red & MD & 2 & 21 \\
\hline Disko & $\mathrm{Fl}$ & Haz. & Seedless & Red & MD & 1 & 21 \\
\hline Dumara & Fl & Sem. & Seeded & Red & WD & 2 & 21 \\
\hline Enchantment & Fl & D. Palm. & Seedless & Red & WD & 1 & 21 \\
\hline Premiere & $\mathrm{Fl}$ & Southw. & Seedless & Red & WD & 2 & 21 \\
\hline Revolution & Fl & Suns. & Seedless & Red & WD & 4 & 21 \\
\hline Stargazer & $\mathrm{Fl}$ & Sem. & Seeded & Red & WD & 3 & 21 \\
\hline Sterling & Fl & Holl. & Seeded & Red & --- & 1 & 21 \\
\hline Summer Sweet 2532 & $\mathrm{Fl}$ & A. \& C. & Seedless & Red & WD & 1 & 21 \\
\hline Summer Sweet 5244 & Fl & A. \& C. & Seedless & Red & WD & 1 & 21 \\
\hline Sweet Eat-N & $\mathrm{Fl}$ & D. Palm. & Seedless & Red & $\mathrm{MD}$ & 2 & 21 \\
\hline Tri-X-Palomar & $\mathrm{Fl}$ & Syng. & Seedless & Red & NR & 5 & 21 \\
\hline Triple Sweet & Fl & Syng. & Seedless & Red & MD & 2 & 21 \\
\hline Afternoon Delight & $\mathrm{Fl}$ & D. Palm. & Seedless & Red & $\mathrm{MD}$ & 2 & 20 \\
\hline Amarillo & $\mathrm{Fl}$ & Syng. & Seedless & Canary yellow & NR & 1 & 20 \\
\hline Butterball & $\mathrm{Fl}$ & D. Palm. & Seedless & Canary yellow & NR & 1 & 20 \\
\hline Buttercup & $\mathrm{Fl}$ & Sutt. & Seedless & Canary yellow & $\mathrm{NR}$ & 2 & 20 \\
\hline Celebration & $\mathrm{Fl}$ & Syng. & Seeded & Red & WD & 3 & 20 \\
\hline Constitution & $\mathrm{Fl}$ & Suns. & Seedless & Red & $\mathrm{MD}$ & 2 & 20 \\
\hline Corporal & Fl & Sem. & Seeded & Red & WD & 1 & 20 \\
\hline Delta & $\mathrm{Fl}$ & Sem. & Seeded & Red & MD & 2 & 20 \\
\hline Falcon & Fl & Sem. & Seeded & Red & WD & 2 & 20 \\
\hline
\end{tabular}

Data for our study and for each trial were analyzed using the MEANS and GLM procedures of SAS-STAT Statistical Software Package (SAS Institute, Cary, N.C.).

\section{Results and discussion}

Rind thickness in the watermelon cultivars tested in this experiment ranged from 7 to $24 \mathrm{~mm}(0.28$ to 0.94 inch), with an average of $18 \mathrm{~mm}$ (0.71 inch). The 50 most suitable cultivars for rind pickle production, group 1 , had rind 20 to $24 \mathrm{~mm}$ thick (Table 1 ).
Another 59 cultivars, group 2, having a rind thickness of 10 to $19 \mathrm{~mm}$, might also be suitable for rind pickle production. The rinds of three cultivars, group 3, were too thin $(<10 \mathrm{~mm})$ to be used in production of rind pickles. Cultivars in groups 1 and 2 should be considered suitable for pickle production with cubes of rind measuring at least $10 \mathrm{~mm}$ per side, as required by many recipes.

Variability of rind thickness among different years of trialing was low (Table 2). Nevertheless, a specific study to

Table 1 continued on next page.

measure the amount of variability on a plot-mean basis, rather than a trialmean basis as we did, could add useful information to our results if someone were interested in running additional experiments in the future.

All nine cultivars that were inbred lines were in groups 1 and 2. Group 1 included 28 seedless and 22 seeded cultivars, while group 2 had 27 seedless and 32 seeded, for a total of 55 seedless 
Table 1. Continued from previous page.

\begin{tabular}{|c|c|c|c|c|c|c|c|}
\hline $\begin{array}{l}\text { Cultivar } \\
\text { name }\end{array}$ & $\begin{array}{c}\text { Cultivar } \\
\text { type }^{\mathrm{z}}\end{array}$ & $\begin{array}{c}\text { Seed } \\
\text { source }^{\mathrm{y}}\end{array}$ & $\begin{array}{l}\text { Fruit } \\
\text { type }\end{array}$ & $\begin{array}{l}\text { Flesh } \\
\text { color }\end{array}$ & $\begin{array}{l}\text { Rind } \\
\text { color }^{\mathrm{x}}\end{array}$ & $\begin{array}{c}\text { Years } \\
\text { of trial }^{\mathrm{w}}\end{array}$ & $\begin{array}{c}\text { Rind } \\
\text { thickness } \\
(\mathrm{mm})^{\mathrm{v}}\end{array}$ \\
\hline Gem-Dandy & $\mathrm{Fl}$ & Will. & Seedless & Red & MD & 4 & 20 \\
\hline Pinata (Large Seeds) & $\mathrm{F} 1$ & Will. & Seedless & Red & WD & 4 & 20 \\
\hline Stars-N-Stripes & $\mathrm{Fl}$ & Sem. & Seeded & Red & MD & 2 & 20 \\
\hline Summer Flavor 900 & Fl & A. \& C. & Seeded & Red & WD & 1 & 20 \\
\hline Sunsation & $\mathrm{Fl}$ & D. Palm. & Seedless & Red & MD & 1 & 20 \\
\hline Sweet Slice & $\mathrm{Fl}$ & Will. & Seedless & Red & MD & 1 & 20 \\
\hline Tastigold & $\mathrm{Fl}$ & Will. & Seeded & Canary yellow & LS & 1 & 20 \\
\hline Wrigley & $\mathrm{Fl}$ & Sem. & Seedless & Red & MD & 1 & 20 \\
\hline Yellow Rose & $\mathrm{Fl}$ & Syng. & Seeded & Canary yellow & MD & 2 & 20 \\
\hline \multicolumn{8}{|c|}{ Group 2: rind thickness $=10-19 \mathrm{~mm}(0.39-0.75 \mathrm{inch})$} \\
\hline Ace of Hearts & $\mathrm{Fl}$ & Sem. & Seedless & Red & WD & 1 & 19 \\
\hline Crimson Sweet & $\mathrm{OP}$ & Will. & Seeded & Red & MD & 5 & 19 \\
\hline Festival & Fl & Will. & Seeded & Red & WD & 4 & 19 \\
\hline Freedom & $\mathrm{Fl}$ & Suns. & Seedless & Red & NR & 5 & 19 \\
\hline Gold Strike & $\mathrm{Fl}$ & Will. & Seeded & Salmon yellow & MD & 1 & 19 \\
\hline Gypsy & $\mathrm{Fl}$ & Sem. & Seedless & Red & MD & 3 & 19 \\
\hline Millionaire & $\mathrm{Fl}$ & H. Mor. & Seedless & Red & MD & 6 & 19 \\
\hline Omega & $\mathrm{Fl}$ & Sem. & Seedless & Red & WD & 1 & 19 \\
\hline Orange Sunshine & $\mathrm{Fl}$ & U.S. Seed. & Seedless & Orange & MD & 2 & 19 \\
\hline Orange Sweet & $\mathrm{Fl}$ & U.S. Seed. & Seedless & Orange & NR & 2 & 19 \\
\hline Orangeglo & $\mathrm{OP}$ & Will. & Seeded & Orange & MD & 1 & 19 \\
\hline Sapphire & $\mathrm{Fl}$ & Holl. & Seedless & Red & --- & 1 & 19 \\
\hline Seedless Sangria & $\mathrm{Fl}$ & Syng. & Seedless & Red & WD & 1 & 19 \\
\hline Sentinel & $\mathrm{Fl}$ & Sem. & Seeded & Red & WD & 3 & 19 \\
\hline Sugar Slice & Fl & Will. & Seedless & Red & MD & 1 & 19 \\
\hline Supersweet 700 & $\mathrm{Fl}$ & A. \& C. & Seeded & Red & NR & 1 & 19 \\
\hline Summer Sweet 5244 & $\mathrm{Fl}$ & A. \& C. & Seedless & Red & MD & 5 & 19 \\
\hline Tri-X-313 & $\mathrm{Fl}$ & Syng. & Seedless & Red & WD & 6 & 19 \\
\hline Boston & $\mathrm{Fl}$ & Suns. & Seedless & Red & NR & 2 & 18 \\
\hline Crimson Trio & $\mathrm{Fl}$ & Syng. & Seedless & Red & NR & 4 & 18 \\
\hline Deuce of Hearts & $\mathrm{Fl}$ & Sem. & Seedless & Red & NR & 1 & 18 \\
\hline Dulce & $\mathrm{Fl}$ & D. Palm. & Seedless & Red & WD & 1 & 18 \\
\hline Genesis & $\mathrm{Fl}$ & Sham. & Seedless & Red & --- & 2 & 18 \\
\hline Jamboree & Fl & Syng. & Seeded & Red & WD & 1 & 18 \\
\hline Lady & $\mathrm{Fl}$ & D. Palm. & Seeded & Red & NR & 1 & 18 \\
\hline Summer Flavor 910 & $\mathrm{Fl}$ & A. \& C. & Seeded & Red & WD & 1 & 18 \\
\hline Super Gold & $\mathrm{Fl}$ & Will. & Seeded & Canary yellow & WD & 2 & 18 \\
\hline Vista & $\mathrm{Fl}$ & Holl. & Seeded & Red & NR & 1 & 18 \\
\hline Big Stripe & $\mathrm{Fl}$ & Will. & Seeded & Red & MD & 2 & 17 \\
\hline Black Diamond & $\mathrm{OP}$ & Will. & Seeded & Red & DS & 2 & 17 \\
\hline Compadre & $\mathrm{Fl}$ & D. Palm. & Seeded & Red & WD & 1 & 17 \\
\hline Fiesta & $\mathrm{Fl}$ & Syng. & Seeded & Red & WD & 7 & 17 \\
\hline Imagination & $\mathrm{Fl}$ & Syng. & Seedless & Red & DS & 1 & 17 \\
\hline Mardi Gras & $\mathrm{Fl}$ & Syng. & Seeded & Red & WD & 5 & 17 \\
\hline Regency & $\mathrm{Fl}$ & Sem. & Seeded & Red & MD & 5 & 17 \\
\hline Seville & $\mathrm{Fl}$ & Holl. & Seeded & Red & $\mathrm{MD}$ & 2 & 17 \\
\hline Summer Gold & Fl & Will. & Seeded & Salmon yellow & MD & 2 & 17 \\
\hline Tendersweet Orange & $\mathrm{OP}$ & Will. & Seeded & Orange & $\mathrm{MD}$ & 2 & 17 \\
\hline Yellow Crimson & $\mathrm{OP}$ & Will. & Seeded & Canary yellow & MD & 1 & 17 \\
\hline AU-Golden Producer & $\mathrm{OP}$ & Will. & Seeded & Salmon yellow & $\mathrm{MD}$ & 1 & 16 \\
\hline Fenway & $\mathrm{F} 1$ & Sem. & Seedless & Red & $\mathrm{MD}$ & 1 & 16 \\
\hline Honey Heart & $\mathrm{Fl}$ & Sem. & Seedless & Canary yellow & --- & 1 & 16 \\
\hline Patriot & $\mathrm{Fl}$ & Will. & Seeded & Red & $\mathrm{MD}$ & 2 & 16 \\
\hline Gold & $\mathrm{Fl}$ & Sham. & Seedless & Canary yellow & --- & 2 & 16 \\
\hline Sugarheart & $\mathrm{Fl}$ & Zer. Ged. & Seedless & Red & $\mathrm{MD}$ & 1 & 16 \\
\hline Triton & Fl & Sem. & Seedless & Canary yellow & NR & 2 & 16 \\
\hline
\end{tabular}

Table 1 continued on next page. 
Table 1. Continued from previous page.

\begin{tabular}{|c|c|c|c|c|c|c|c|}
\hline $\begin{array}{l}\text { Cultivar } \\
\text { name }\end{array}$ & $\begin{array}{c}\text { Cultivar } \\
\text { type }^{\mathrm{z}}\end{array}$ & $\begin{array}{c}\text { Seed } \\
\text { source }^{\mathrm{y}}\end{array}$ & $\begin{array}{c}\text { Fruit } \\
\text { type }\end{array}$ & $\begin{array}{l}\text { Flesh } \\
\text { color }\end{array}$ & $\begin{array}{l}\text { Rind } \\
\text { color }^{\mathrm{x}}\end{array}$ & $\begin{array}{c}\text { Years } \\
\text { of trial }^{w}\end{array}$ & $\begin{array}{c}\text { Rind } \\
\text { thickness } \\
(\mathrm{mm})^{\mathrm{v}}\end{array}$ \\
\hline Tri-X-Shadow & $\mathrm{Fl}$ & Syng. & Seedless & Red & MS & 5 & 16 \\
\hline Desert Storm & Fl & Will. & Seeded & Red & WD & 2 & 15 \\
\hline Millenium & $\mathrm{Fl}$ & H. Mor. & Seedless & Red & NR & 5 & 15 \\
\hline Sultan & $\mathrm{Fl}$ & H. Mor. & Seeded & Red & WD & 2 & 15 \\
\hline Plantation Pride & $\mathrm{Fl}$ & D. Palm. & Seeded & Red & WD & 1 & 14 \\
\hline Baron & $\mathrm{Fl}$ & Syng. & Seeded & Red & MS & 2 & 13 \\
\hline Ole’ & $\mathrm{Fl}$ & Will. & Seeded & Red & WD & 1 & 13 \\
\hline Royal Flush & $\mathrm{Fl}$ & Sem. & Seeded & Red & WD & 2 & 13 \\
\hline Yellow Doll & Fl & Sem. & Seeded & Canary yellow & NR & 2 & 11 \\
\hline \multicolumn{8}{|c|}{ Group 3: rind thickness $<10 \mathrm{~mm}$ (0.39 inch) } \\
\hline Scarlet Trio & Fl & Syng. & Seedless & Red & NR & 1 & 8 \\
\hline \multirow[t]{2}{*}{ Carnival } & $\mathrm{Fl}$ & Syng. & Seeded & Red & MD & 1 & 7 \\
\hline & & & Statistic & & & & \\
\hline $\operatorname{LSD}^{\mathrm{u}}$ & & & & & & & 4 \\
\hline \multirow[t]{5}{*}{ Mean } & Overall & & & & & & 18 \\
\hline & Seedless & & & & & & 19 \\
\hline & Seeded & & & & & & 18 \\
\hline & Red flesh & & & & & & 19 \\
\hline & Orange/yellow & & & & & & 8 \\
\hline Maximum & & & & & & & 24 \\
\hline Minimum & & & & & & & 7 \\
\hline
\end{tabular}

${ }^{\mathrm{z}} \mathrm{F}_{1}=$ hybrid cultivar; $\mathrm{OP}=$ inbred cultivar.

${ }^{y}$ A. \& C. = Abbott \& Cobb, Feasterville, Pa.; D. Palm. = D. Palmer Seed Co.,Yuma, Ariz.; H. Mor. = Harris Moran, Modesto, Calif.; Haz. = Hazera Seeds, El Segundo, Calif.; Holl. = Hollar Seeds, Rocky Ford, Colo.; John. = Johnny's Selected Seeds, Winslow, Maine; Seedw. = Seedway, Hall, N.Y.; Sem. = Seminis, Oxnard, Calif.; Sham. = Shamrock Seed Co., Salinas, Calif.; Southw. = Southwestern Vegetable Seed Co., Casa Grande, Ariz.; Suns. = Sunseeds, Parma, Idaho; Sutt. = Sutter Seeds, Yuba City, Calif.; Syng. = Syngenta Seeds, Golden Valley, Minn.; U.S. Seed. = U.S. Seedless, Falls Church, Va.; Uni. Gen. = United Genetics, Hollister, Calif.; Will. = Willhite Seed, Poolville, Texas; Zer. Ged. = Zeraim Gedera, Gedera, Israel.

${ }^{x} \mathrm{NR}, \mathrm{MD}, \mathrm{WD}=$ narrow, medium, and dark green stripes on light green background, respectively; GR = gray; LS, MS, DS = light, medium, and dark solid green, respectively. "Each year of trial was a randomized complete-block design with one location in North Carolina (Clayton, Clinton, Kinston, or Chowan Co.), 1 year (1995-2002), and three replications.

"Rind thickness was measured on mature fruit in millimeters from the outer edge of the fruit to the boundary between white mesocarp and colored endocarp for each harvest up to five fruit per plot $(25.4 \mathrm{~mm}=1 \mathrm{inch})$; fruit were cut longitudinally and rind thickness measured at mid-length.

"LSD $=$ Fisher's protected least significant difference $(\alpha=0.05)$.

and 54 seeded watermelon cultivars suitable for industrial production of rind pickles. Group 3 included one seedless and two seeded cultivars.

All cultivars with yellow or orange flesh were classified in groups 1 and 2. Therefore, growers and processors interested in watermelons with yellow or orange flesh for fresh fruit salads can use the rinds of these cultivars in the production of rind pickles. Nevertheless, according to the estimated LSD for the experiment, 'Desert King' was the only cultivar with non-red flesh color among those having the thickest rind.

The absence of appreciable differences in rind thickness among the different types of watermelon considered in this study (inbred vs. hybrid, seedless vs. seeded, and red vs. yellow/orange

Table 2. Variability over 5 years of trialing of rind thickness for four watermelon cultivars evaluated in North Carolina in replicated trials conducted between 1995 and 2002.

\begin{tabular}{lcccccc}
\hline $\begin{array}{l}\text { Cultivar } \\
\text { name }\end{array}$ & $\begin{array}{c}\text { Year 1 } \\
\left(\mathbf{m m}^{\mathbf{z}}\right)\end{array}$ & $\begin{array}{c}\text { Year 2 } \\
\left(\mathbf{m m}^{\mathbf{z}}\right)\end{array}$ & $\begin{array}{c}\text { Year 3 } \\
\left(\mathbf{m m}^{\mathbf{z}}\right)\end{array}$ & $\begin{array}{c}\text { Year 4 } \\
\left(\mathbf{m m}^{\mathbf{z}}\right)\end{array}$ & $\begin{array}{c}\text { Year 5 } \\
\left(\mathbf{m m}^{\mathbf{z}}\right)\end{array}$ & $\begin{array}{c}\text { Thickness } \\
\text { group }^{\mathrm{y}}\end{array}$ \\
\hline Tri-X-Palomar & 26 & 22 & 21 & 19 & 18 & 1 \\
Starbrite & 22 & --- & 21 & 27 & 19 & 1 \\
Tri-X-313 & 22 & 19 & 22 & 23 & 19 & 2 \\
Freedom & 17 & 22 & 21 & 22 & 15 & 2 \\
\hline
\end{tabular}

z25.4 mm = 1 inch

${ }^{y}$ Group 1: rind thickness $>19 \mathrm{~mm}$; group 2: rind thickness $=10-19 \mathrm{~mm}$; group 3: rind thickness $<10 \mathrm{~mm}$.

flesh) might be largely due to common standards for selection adopted by watermelon breeders. Objectives usually include thin rind, with the lower limit being the protection of the fruit during shipping. By analyzing this large set of cultivars, we could argue that selection has narrowed the range of rind thick- ness in watermelon between 10 and $20 \mathrm{~mm}$. Recently, new cultivars of very small size [personal size watermelons, having 1.8 to $3.6-\mathrm{kg}$ ( 4 to $8 \mathrm{lb}$ ) fruit] have been introduced, with very tough and thin rind. Although we did not include this new type of watermelon in our study, it is likely that the thin rind 
of most of these cultivars would not be suitable for pickle production.

In conclusion, most of the watermelon cultivars in current production in the U.S. would be good candidates for production of rind pickles. Growers and processors should choose cultivars from groups 1 and 2 of our list, according to their primary needs. Traits such as yield, fruit quality, fruit type, adaptability, and disease resistance should be the main concern when choosing cultivars for field production.

\section{Literature cited}

Abd el Hafez, A.A. 1982. Effect of ploidy differences on fruit characteristics in watermelon. Acta Agron. Acad. Scientiarum Hungaricae 31:66-70.

Gusmini, G. and T.C. Wehner. 2003a. All about watermelon-Watermelon rind pickles. 29 Nov. 2003. <http://cuke.hort. ncsu.edu/cucurbit/wmelon/wmhndbk/ wmrindpickle.html>.

Gusmini, G. and T.C. Wehner. 2003b. Cultivars for watermelon rind pickles. HortScience 38:783. (Abstr.)

Gusmini, G. and T.C. Wehner. 2004. Cultivar suitable for watermelon rind pickles. Cucurbit Genet. Coop. Rpt. (In press.)

Maynard, D.N. (ed.). 2001. Watermelons. Characteristics, production, and marketing. ASHS Press, Alexandria, Va.

Sanders, D.C. (ed.). 2004. Vegetable crop guidelines for the southeastern U.S. 2004-2005. North Carolina Veg. Growers Assn., Raleigh, N.C.

Schultheis, J.R. 1995. Watermelon research and extension report. Hort. Res. Ser. N.C. State Univ., Dept. of Hort. Sci. 119:1-56.

Schultheis, J.R. 1996. Watermelon research and extension report. Hort. Res. Ser. N.C. State Univ., Dept. of Hort. Sci. 120:1-50.
Schultheis, J.R. 1997. Watermelon research and extension report. Hort. Res. Ser. N.C. State Univ., Dept. of Hort. Sci. $121: 1-50$

Schultheis, J.R. 1998. Watermelon research and extension report. Hort. Res. Ser. N.C. State Univ., Dept. of Hort. Sci. 135:1-50.

Schultheis, J.R. 1999. Watermelon research and extension report. Hort. Res. Ser. N.C. State Univ., Dept. of Hort. Sci. 138:1-50.

Schultheis, J.R. 2000. Cucurbit and sweet corn cultivar evaluations. Hort. Res. Ser. N.C. State Univ., Dept. of Hort. Sci. 149:1-50.

Schultheis, J.R. 2001. Cucurbit and sweet corn cultivar evaluations. Hort. Res. Ser. N.C. State Univ., Dept. of Hort. Sci. $152: 1-50$

Schultheis, J.R. 2002. Cucurbit and sweet corn cultivar evaluations. Hort. Res. Ser. N.C. State Univ., Dept. of Hort. Sci. 155:1-50.

Sharma, R.R. and B. Choudhury. 1988. Studies on some quantitative characters in watermelon (Citrullus lanatus Thunb. Mansf.). II. Inheritance of total soluble solids and rind thickness. Indian J. Hort. 45:283-287.

Simonne, A., M. Carter, R. Fellers, J. Weese, C. Wei, E. Simonne, and M.E. Miller. 2003. Chemical, physical and sensory characterization of watermelon rind pickles. J. Food Processing and Preservation 26:415-432.

U.S. Dept. of Agriculture-Agricultural Research Service. 2004. Agricultural statistics database QuickSTAT. 5 Jan. 2004. <http://www.nass.usda.gov:81/ ipedb/>. 